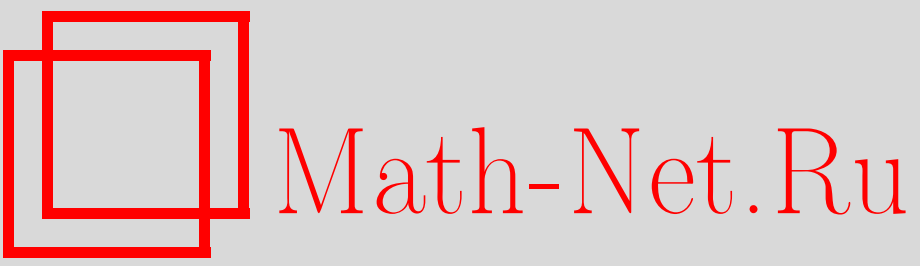

Я. Е. Ромм, Г. А. Джанунц, Варьируемое кусочно-интерполяционное решение уравнения переноса, Итоги науки и техн. Сер. Соврем. мат. и ее прил. Темат. обз., 2019, том 166, 77-86

DOI: https://doi.org/10.36535/0233-6723-2019-166-77-86

Использование Общероссийского математического портала Math-Net.Ru подразумевает, что вы прочитали и согласны с пользовательским соглашением

http: //www.mathnet.ru/rus/agreement

Параметры загрузки:

IP: 3.91 .87 .62

26 апреля 2023 г., 18:30:50 


\title{
ВАРЬИРУЕМОЕ КУСОЧНО-ИНТЕРПОЛЯЦИОННОЕ РЕШЕНИЕ УРАВНЕНИЯ ПЕРЕНОСА
}

\author{
(c) 2019 г. $\quad$ Я. Е. POMМ, Г. А. ДЖАНУНЦ
}

\begin{abstract}
АннотАция. Строится кусочно-интерполяционный метод приближенного решения уравнения переноса на основе интерполяционного полинома Ньютона от двух переменных. Полином преобразуется к алгебраической форме с числовыми коэффициентами, что приводит к последовательности итераций для повышения точности приближения. Метод реализован программно, выполнен численный эксперимент, обсуждается возможность обобщений на системы уравнений в частных производных и интегро-дифференциальные уравнения.
\end{abstract}

Ключевъе слова: кусочно интерполяционная аппроксимация, интерполяционный полином Ньютона для функции двух переменных, задача Коши для уравнений в частных производных, уравнение переноса.

\section{VARIABLE PIECEWISE INTERPOLATION SOLUTION OF THE TRANSPORT EQUATION}

\author{
(c) 2019 YA. E. ROMM, G. A. DZHANUNTS
}

\begin{abstract}
In this paper, we construct a piecewise interpolation method of approximate solution of the transport equation based on the Newton interpolation polynomial of two variables. We transform the polynomial to the algebraic form with numerical coefficients; this leads us to a sequence of iterations, which improves the accuracy of the approximation. The method is implemented in software and numerical experiments ares performed. The possibility of generalizations to systems of partial differential equations and integro-differential equations is discussed.
\end{abstract}

Keywords and phrases: piecewise interpolation approximation, Newton interpolation polynomial for a function of two variables, Cauchy problem for partial differential equations, transport equation.

AMS Subject Classification: 65M12, 65M15, 65D05

1. Введение. Постановка вопроса. Для дифференциальных уравнений в частных производных актуальна проблема снижения погрешности приближенного решения задачи Коши (см. [6,13]). Возможность повышения точности, в частности, при решении уравнения переноса, обсуждается в $[5,9,14,16,19]$. Как правило, границы погрешности существующих методов находятся в диапазоне $10^{-7}-10^{-3}$ (см. [8]). Ниже излагается кусочно-интерполяционное решение задачи Коши для уравнения переноса с итерационным уточнением. Аналогом является метод решения обыкновенных дифференциальных уравнений (см. [4]). Для уравнений в частных производных метод строится на основе интерполяционного полинома Ньютона от двух переменных. Для линейного уравнения переноса доказывается равномерная сходимость, оценивается скорость

Работа выполнена при поддержке Российского фонда фундаментальных исследований (проект № 16-07-00100-а). 
сходимости, для нелинейного уравнения возможны аналогичные оценки. В [12] представлена программа и численный эксперимент, где рассматриваемая задача в области $[0,1] \times[0,1]$ решается с погрешностью $10^{-18}$.

2. Кусочно-интерполяционное вычисление функций двух переменных. Рассматривается система декартовых координат $U X T$ в $\mathbb{R}_{3}$. Метод опирается на кусочную интерполяцию действительной функции $u=u(x, t)$ двух действительных переменных в области

$$
G=\{(x, t) \mid x \in[a, b], t \in[c, d]\} .
$$

Область разбивается на прямоугольные подобласти $G_{i j}$ с пересекающимися границами:

$$
G=\bigcup_{j=0}^{P_{t}-1} \bigcup_{i=0}^{P_{x}-1} G_{i j}
$$

где

$$
G_{i j}=\left\{(x, t) \mid x \in\left[x_{i}, x_{i+1}\right], t \in\left[t_{j}, t_{j+1}\right]\right\}, \quad P_{x}=2^{k_{x}}, \quad P_{t}=2^{k_{t}}, \quad k_{x}, k_{t} \in\{0,1, \ldots\} .
$$

Построение интерполяционного полинома Ньютона от двух переменных в подобласти (3) использует $(n+1)(n+2) / 2$ треугольно расположенных узлов (см. [1]):

$$
\begin{aligned}
& \left(x_{i 0}, t_{j n}\right) \\
& \left(x_{i 0}, t_{j(n-1)}\right) \quad\left(x_{i 1}, t_{j(n-1)}\right) \\
& \ldots \ldots \ldots \ldots \\
& \begin{array}{llll}
\left(x_{i 0}, t_{j 1}\right) \quad\left(x_{i 1}, t_{j 1}\right) & \ldots \ldots \ldots & \left(x_{i(n-1)}, t_{j 1}\right)
\end{array}
\end{aligned}
$$

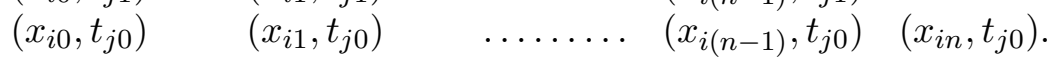

При расположении узлов (4) функция интерполируется в нижней треугольной части области $G_{i j}$; в верхней части требуется экстраполяция. Пусть произвольно задана граница $\varepsilon$ абсолютной погрешности приближения функции $u(x, t)$. В каждой подобласти $G_{i j}, i=\overline{0, P_{x}-1}, j=\overline{0, P_{t}-1}$, интерполяционный полином Ньютона $\Psi_{n}^{i j}(z, w)$ строится с равно отстоящими по направлениям координат узлами $x_{i \ell}, t_{j m}$. Определяются шаги интерполяции и переменные:

$$
h_{x}=\frac{x_{i+1}-x_{i}}{n}, \quad h_{t}=\frac{t_{j+1}-t_{j}}{n}, \quad z=\frac{x-x_{i}}{h_{x}}, \quad w=\frac{t-t_{j}}{h_{t}},
$$

где $(x, t) \in G_{i j}($ см. $(3))$. Пусть $\left(x_{i \ell}, t_{j m}\right)$ - узел интерполяции с координатами

$$
x_{i \ell}=x_{i}+\ell h_{x}, \quad t_{j m}=t_{j}+m h_{t}, \quad \ell=\overline{0, n}, \quad m=\overline{0, n-\ell} .
$$

Интерполяционный полином Ньютона примет вид

$$
\Psi_{n}^{i j}(z, w)=u\left(x_{i 0}, t_{j 0}\right)+\sum_{k=1}^{n} \sum_{s=0}^{k} \frac{\Delta_{x^{s} t^{k-s}}^{k} u\left(x_{i 0}, t_{j 0}\right)}{s !(k-s) !} \prod_{\ell=0}^{s-1}(z-\ell) \prod_{m=0}^{k-s-1}(w-m) .
$$

В (7) величины $\Delta_{x^{s} t^{k-s}}^{k} u\left(x_{i 0}, t_{j 0}\right)$ - конечные разности $k$-го порядка, которые при $k=\overline{1, n}$, $s=\overline{0, k}$ с учетом (6) вычисляются из соотношений

$$
\left\{\begin{array}{l}
\Delta_{x^{1} t^{0}} u\left(x_{i \ell}, t_{j m}\right)=u\left(x_{i(\ell+1)}, t_{j m}\right)-u\left(x_{i \ell}, t_{j m}\right), \\
\Delta_{x^{0} t^{1}} u\left(x_{i \ell}, t_{j m}\right)=u\left(x_{i \ell}, t_{j(m+1)}\right)-u\left(x_{i \ell}, t_{j m}\right), \\
\Delta_{x^{s} t^{k-s}}^{k} u\left(x_{i \ell}, t_{j m}\right)=\Delta_{x^{s} t^{k-s-1}}^{k-1} u\left(x_{i \ell}, t_{j(m+1)}\right)-\Delta_{x^{s} t^{k-s-1}}^{k-1} u\left(x_{i \ell}, t_{j m}\right) \quad \text { при } k>s, \\
\Delta_{x^{s} t^{k-s}}^{k} u\left(x_{i \ell}, t_{j m}\right)=\Delta_{x^{s-1} t^{k-s}}^{k-1} u\left(x_{i(\ell+1)}, t_{j m}\right)-\Delta_{x^{s-1} t^{k-s}}^{k-1} u\left(x_{i \ell}, t_{j m}\right) \quad \text { при } k=s,
\end{array}\right.
$$

где $k=\overline{2, n}, s=\overline{0, k}, \ell=\overline{0, n-k}, m=\overline{0, n-k-\ell}$. Для заданного $\varepsilon>0$ степень полинома $n$ выбирается одинаковой во всех подобластях и минимальной при условии

$$
\left|u(x, t)-\Psi_{n}^{i j}(z, w)\right| \leqslant \varepsilon \quad \forall(x, t) \in G_{i j}, \quad i=\overline{0, P_{x}-1}, \quad j=\overline{0, P_{t}-1} .
$$

Проверка точности приближения (9) в каждой подобласти проводится дискретно на множестве проверочных точек $\left(x_{i s}, t_{j r}\right)$, равноотстоящих на величины $h_{x} / \gamma$ и $h_{t} / \gamma$ по направлению каждой 
координаты, $x_{i s}=x_{i}+s h_{x} / \gamma, t_{j r}=t_{j}+r h_{t} / \gamma, s, r \in\{0,1, \ldots\}$, где $\gamma \geqslant 3$ - параметр. Минимальность степени $n$ обеспечивается следующим образом. Построение и проверка точности начинается от $n=1$ и $k_{x}=0, k_{t}=0$ во всех подобластях из (1)-(3). При нарушении неравенства (9) хотя бы в одной проверочной точке какой-либо подобласти значения $k_{x}$ и $k_{t}$ увеличиваются на единицу, и проверка начинается сначала. Так продолжается до нарушения априори заданной границы возрастания $k_{x}$ и $k_{t}: k_{x} \leqslant k_{0}, k_{t} \leqslant k_{0}$. Если в результате заданная точность приближения не достигнута, то полагается $k_{x}=0, k_{t}=0$, при этом степень $n$ увеличивается на единицу и проверка возобновляется сначала для этой зафиксированной степени в тех же границах $k_{x}$ и $k_{t}$. Так продолжается до нарушения априори заданной границы возрастания $n: n \leqslant N_{0}$. В качестве искомого фиксируется наименьшее $n$, при котором неравенство (9) выполняется одновременно во всех проверочных точках всех $2^{k_{x}+k_{t}}$ подобластей, в соответствии с этим фиксируются текущие значения $k_{x}$ и $k_{t}$. Ниже предполагается, что степень $n$ ограничена, в то время как количество подобластей в (1)-(3) не ограничивается: $n \leqslant N_{0}, N_{0}=$ const; $k_{x} \rightarrow \infty, k_{t} \rightarrow \infty$. Полином (7) с использованием видоизмененных формул Виета (см. [10]) преобразуется к виду полинома с числовыми коэффициентами:

$$
\Psi_{n}^{i j}(z, w)=\sum_{\ell=0}^{n} \sum_{m=0}^{n-\ell} a_{\ell m}^{i j} z^{\ell} w^{m}
$$

Значение (10) вычисляется с помощью аналога схемы Горнера:

$$
\begin{aligned}
\Psi_{n}^{i j}(z, w)=\left(\ldots \left[\left(a_{0 n}^{i j} w+a_{1 n-1}^{i j} z\right.\right.\right. & \left.+a_{0 n-1}^{i j}\right) w+ \\
& \left.\left.\quad+\left(a_{2 n-2}^{i j} z+a_{1 n-2}^{i j}\right) z+a_{0 n-2}^{i j}\right] w+\ldots\right) w+\cdots+a_{00}^{i j} .
\end{aligned}
$$

Для аппроксимации $(9)$ функции $u(x, t),(x, t) \in G$, дешифруются индексы подобласти $G_{i j}$ :

$$
i=\operatorname{int} \frac{x-a}{\rho_{x}}, \quad j=\operatorname{int} \frac{t-c}{\rho_{t}},
$$

int - целая часть числа, $\rho_{x}=x_{i+1}-x_{i}, \rho_{t}=t_{j+1}-t_{j}, i=\overline{0, P_{x}-1}, j=\overline{0, P_{t}-1}, x \in\left[x_{i}, x_{i+1}\right), t \in$ $\left[t_{j}, t_{j+1}\right)$. Индексы служат математическим адресом массива коэффициентов полинома в памяти компьютера. Код программы, выполняющей описанную аппроксимацию, приводится в [2, 3, 11]. Для оценки погрешности кусочной интерполяции полином (7) рассматривается в эквивалентной форме:

$$
\Psi_{n}^{i j}(x, t)=u\left(x_{i 0}, t_{j 0}\right)+\sum_{m=1}^{n} \sum_{k=0}^{m} \frac{\Delta_{x^{k} t^{m-k}}^{m} u\left(x_{i 0}, t_{j 0}\right)}{h_{x}^{k} h_{t}^{m-k} k !(m-k) !} \prod_{q=0}^{k-1}\left(x-x_{i q}\right) \prod_{r=0}^{m-k-1}\left(t-t_{j r}\right) .
$$

Соответствие между (11) и (7) реализуется заменами $z=\left(x-x_{i 0}\right) / h_{x}, w=\left(t-t_{j 0}\right) / h_{t}$. Пусть вначале полином (11) построен для интерполяции функции $u(x, t)$ во всей области $G$ из $(1)-(3)$. В этом случае для простоты не используется индексация подобласти. Остаточный член интерполяции можно представить в следующем виде (см. $[17,18])$ :

$$
R_{G}(x, t)=\sum_{i=0}^{n+1} \frac{\partial^{n+1} u\left(\xi_{i}, \eta_{i}\right)}{\partial x^{i} \partial t^{n+1-i}} \frac{1}{i !} \prod_{\ell=0}^{i-1}\left(x-x_{\ell}\right) \frac{1}{(n-i+1) !} \prod_{k=0}^{n-i}\left(t-t_{k}\right),
$$

где $\left(x_{\ell}, t_{k}\right)$ - узел интерполяции, $\left(\xi_{i}, \eta_{i}\right)$ - некоторая точка внутри области $G$. Внутри области производные понимаются в обычном смысле, на границе - как односторонние производные по направлению изнутри к границе. Вводится предположение о существовании, непрерывности, и, следовательно, ограниченности в прямоугольнике $G$ всех смешанных производных до порядка $2 n+1$ включительно. В (11) достаточно было бы такое предположение относительно порядка $n+1$. Смысл формального завышения порядка гладкости состоит в следующем. В силу треугольности расположения узлов (4) остаточный член (12) фактически относится только к нижней треугольной части, а не ко всей прямоугольной области $G$. Для оценки погрешности преобразуется 
расположение узлов. Именно, интерполяционный полином Ньютона всегда будет строиться в описанном прямоугольном треугольнике, катеты которого продолжают нижнюю и левую стороны прямоугольной области $G$, длина каждого катета вдвое больше продолжаемой стороны. Гипотенуза треугольника, охватывающего все узлы, пройдет через правую вершину прямоугольной области $G$. Очевидно, узлы (4) в данном треугольнике заведомо охватят всю область $G$, и остаточный член (12) будет относиться уже ко всей этой области. Аналогично, когда полином (11) будет строиться для прямоугольной подобласти $G_{i j}$, узлы интерполяции будут располагаться в описанном прямоугольном треугольнике, охватывающем $G_{i j}$. Согласно этому преобразованию расстояния между узлами интерполяции $h_{x}, h_{t}$ в $(5)$, при неизменности степени полинома $n$, окажутся вдвое больше первоначально предполагавшихся в (5) значений. Чтобы не росла погрешность интерполирования, расстояния будут сохранены такими, как они даны в (5). Но тогда степень интерполирующего полинома увеличится вдвое и станет равной $2 n$ в соответствии с количеством $(2 n+1)(2 n+2) / 2$ узлов и их преобразованным расположением. Это изменение степени будет учитываться в дальнейших оценках, где $n$ всюду заменяется на $2 n$. В [3] показано, что при данных предположениях

$$
\left|R_{G}(x, t)\right| \leqslant \sum_{i=0}^{2 n+1}\left|\frac{\partial^{2 n+1} u\left(\xi_{i}, \eta_{i}\right)}{\partial x^{i} \partial t^{2 n+1-i}}\right| h_{x}^{i} h_{t}^{2 n-i+1}
$$

где

$$
\max _{G}\left|\frac{\partial^{2 n+1} u(x, t)}{\partial x^{i} \partial t^{2 n+1-i}}\right|=c, \quad c=\text { const }, c<\infty, \quad \forall i \leqslant 2 n+1, \forall n \leqslant N_{0} .
$$

Отсюда

$$
\left|R_{G}(x, t)\right| \leqslant c \sum_{i=0}^{2 n+1} h_{x}^{i} h_{t}^{2 n-i+1} .
$$

Ниже предполагается, что область $G$ такова, что расстояния между узлами меньше единицы. Поскольку узлы являются равноотстоящими вдоль направления осей, то найдутся такие $h, q, p$, что

$$
h_{x}=q h, \quad q=\mathrm{const}, \quad h_{t}=p h, \quad p=\mathrm{const}, \quad h<1, q<1, p<1, p<q .
$$

С учетом (16) оценка (15) перейдет в соотношение

$$
\left|R_{G}(x, t)\right| \leqslant c \sum_{i=0}^{2 n+1}(q h)^{i}(p h)^{2 n-i+1}=c h^{2 n+1} \sum_{i=0}^{2 n+1} q^{i} p^{2 n-i+1} .
$$

Отсюда и из (16)

$$
\left|R_{G}(x, t)\right| \leqslant C h^{2 n+1} ; \quad C=c\left(-e \log _{q} e\right), \quad C=\text { const } .
$$

В (13) величины $h_{x}, h_{t}$ должны быть соответственно пропорционально уменьшены до рассматриваемых размеров. Получится:

$$
\left|R_{G_{i j}}(x, t)\right| \leqslant c \sum_{i=0}^{2 n+1} \frac{h_{x}^{i}}{2^{i k_{x}}} \frac{h_{t}^{2 n-i+1}}{2^{(2 n-i+1) k_{t}}}
$$

где $c$ такое же, как в (14). Если $2^{k_{x}}=2^{k_{t}}=2^{k}$, то $h$ в (16) уменьшится в обратной пропорции $2^{k}$. В этом случае

$$
\left|R_{G_{i j}}(x, t)\right| \leqslant C \frac{h^{2 n+1}}{2^{k(2 n+1)}}
$$

где $C$ из $(17),(x, t) \in G_{i j}$, а $G_{i j}$ - из (2). Из изложенного вытекает следующее утверждение.

Лемма 1. В области (1), объединяющей $2^{2 k}$ подобластей (2), (3), при условии существования и непрерывности всех смешанных производных функции $u(x, t)$ до порядка $2 n+1$ включительно, 
кусочно-интерполячионное приближение (9) полиномами в виде (10), а также (11) выполняется с абсолютной погрешностью

$$
O\left(\frac{h^{2 n+1}}{2^{k(2 n+1)}}\right), \quad h<1 .
$$

С учетом (10) имеют место соотношения:

$$
\begin{aligned}
u(x, t) \approx & \Psi_{2 n}^{i j}(z, w)=\sum_{\ell=0}^{2 n} \sum_{m=0}^{2 n-\ell} a_{\ell m} z^{\ell} w^{m} ; \quad z=\frac{x-x_{i 0}}{h_{x}}, \quad w=\frac{t-t_{j 0}}{h_{t}} ; \\
u_{x} \approx & \frac{1}{h_{x}} \sum_{\ell=1}^{2 n} \sum_{m=0}^{2 n-\ell} \ell a_{\ell m} z^{\ell-1} w^{m} ; \quad u_{t} \approx \frac{1}{h_{t}} \sum_{\ell=0}^{2 n} \sum_{m=1}^{2 n-\ell} m a_{\ell m} z^{\ell} w^{m-1} \\
& \int u(x, t) d t \approx \tilde{c}+h_{t} \sum_{\ell=0}^{2 n} \sum_{m=0}^{2 n-\ell} a_{\ell m} z^{\ell} \frac{w^{m+1}}{m+1} ; \quad \tilde{c}=\mathrm{const} .
\end{aligned}
$$

Значения констант и параметров оговариваются в дальнейшем, при интерполировании решения уравнений в частных производных будет изменено обозначение полинома из (19), что также оговаривается по ходу изложения. Частную производную по времени приближают согласно (19), а решение восстанавливается путем взятия первообразной.

3. Кусочно-интерполяционное решение задачи Коши для линейного уравнения переноса. Рассматривается задача вида

$$
\frac{\partial u}{\partial t}+a(x, t) \frac{\partial u}{\partial x}=f(x, t) ; \quad u(x, 0)=\varphi(x)
$$

(см. [7,15]), где $a, f$-заданные функции независимых переменных $x$ и $t$ в полуплоскости $\{(x, t) \mid$ $x \in \mathbb{R}, t \geqslant 0\}, \varphi$ - заданная функция переменной $x$. Используются следующие предположения.

I. Приближенное решение задачи (22) требуется построить в прямоугольной области $G$ из (1), разбиваемой на подобласти (2), (3). Считается, что в (1) $c=0, d=T, t \in[0, T]$.

II. Предполагается, что в области $\{(x, t) \mid x \in \mathbb{R}, t \geqslant 0\}$ (в частности, в $G$ ) выполнены все условия существования и единственности.

III. Предполагается, что решение $u_{h}$ разностной схемы с равномерной прямоугольной сеткой, построенной для задачи $(22)$, сходится к решению $u(x, t)$ с оценкой

$$
\left\|u(x, t)-u_{\tilde{h}}\right\|=O\left(\tau^{p_{0}}+\tilde{h}^{q_{0}}\right), \quad 1 \leqslant p_{0}, \quad 1 \leqslant q_{0},
$$

где $\tilde{h}$ и $\tau$ - шаги сетки вдоль $x$ и $t,\left\|u(x, t)-u_{\tilde{h}}\right\|$ - сеточная норма, определяемая как максимальное значение проекции $\left|u(x, t)-u_{\tilde{h}}\right|$ на равномерную сетку.

IV. Предполагается существование и непрерывность в этой области смешанных производных решения $u(x, t)$ до порядка $2 n+1$ включительно.

В предположении IV при интерполяции решения полиномом вида (19) будет выполнена оценка (18). Приближение решения задачи (22) с помощью интерполяционного полинома Ньютона строится в каждой подобласти $G_{i j}$ с последовательным переходом от текущей подобласти к подобласти смежной с ней. При таком переходе значения полинома на границе $G_{i j}$ определяют начальные условия для перехода в смежную подобласть $G_{i j+1}$. Для начального приближения решения в данном процессе в качестве исходных узловых значений интерполяции используются значения функции $\varphi(x)$ из (22). На этой основе с использованием (5)-(11), а также (20) и (21) строится интерполяционное приближение производной

$$
\frac{\partial u}{\partial t}=f(x, t)-a(x, t) \frac{\partial u}{\partial x}
$$

Интеграл по времени от полученного полинома, при условии подстановки производной функции $\partial \varphi / \partial x$ в качестве приближенного значения $\partial u / \partial x$, принимается за новое приближение решения. 
Согласно (22) условие $\forall(x, t) \in G_{i j}$ выполнено:

$$
\int_{t_{0}}^{t} \frac{\partial u(x, t)}{\partial t} d t=\int_{t_{0}}^{t}\left(f(x, t)-a(x, t) \frac{\partial u(x, t)}{\partial x}\right) d t, \quad t_{0}=t_{j 0}
$$

К обозначению полинома $\Psi_{2 n}^{i j}(z, w)$ из (19) добавляется нижний индекс $2 k$ в соответствии с числом подобластей $2^{2 k}$, и аналогичная индексация применяется к другим компонентам рассматриваемых величин и функций. Полином $\Psi_{2 k 2 n}^{i j}(z, w)$ будет приближать не решение, а подынтегральную функцию правой части (23) с остаточным членом $c_{2 k, 2 n}$, оцениваемым при помощи (18):

$$
\Psi_{2 k 2 n}^{i j}(z, w)=f(x, t)-a(x, t) \frac{\partial \tilde{u}}{\partial x}+c_{2 k, 2 n},
$$

где $\tilde{u}$ - приближение решения: $\tilde{u}(x, t) \approx u(x, t)$. При этом, как и полином из (19), полином в левой части (24) переводится в форму с числовыми коэффициентами, аналогичную (10). Полученный таким образом полином обозначается ${ }^{t} P_{u, 2 k, 2 n}(x, t)$ - по соответствию рассматриваемой степени $2 n$, количеству подобластей $2^{2 k}$ и приближению решения путем интегрирования по $t$ из (23). Остаточный член приближения от этого полинома обозначается $c_{u, 2 k, 2 n}$ и оценивается с учетом (24) из соотношения

$$
{ }^{t} P_{u, 2 k, 2 n}(x, t)={ }^{t} P_{u, 2 k, 2 n}\left(x, t_{0}\right)+\int_{t_{0}}^{t}\left(f(x, t)-a(x, t) \frac{\partial \tilde{u}(x, t)}{\partial x}\right) d t+{ }^{t} c_{u, 2 k, 2 n},{ }^{t} c_{u, 2 k, 2 n}=\int_{t_{0}}^{t} c_{2 k, 2 n} d t,
$$

где

$$
\left|{ }^{t} c_{u, 2 k, 2 n}\right| \leqslant \tilde{C} \frac{h^{2 n+1}}{2^{k(2 n+2)}}, \quad \tilde{C}=\text { const } \quad \forall(x, t) \in G_{i j}, \quad \forall \forall G_{i j} \in G .
$$

В [12] доказано следующее утверждение.

Теорема 1. В условиях I-IV интерполяиионный полином ${ }^{t} P_{u, 2 k, 2 n}(x, t)$ в каждой подобласти $G_{i j}$ равномерно сходится к решению задачи $(22)$ при $k \rightarrow \infty$, где $2^{2 k}-$ число подобластей. Равномерная сходимость достигается во всей области $G$, если интерполяиия выполняется в каждой подобласти, скорость сходимости оченивается из соотношения

$$
\left|{ }^{t} P_{u, 2 k, 2 n}(x, t)-u(x, t)\right| \leqslant \tilde{C} \frac{h^{2 n+1}}{2^{k \times 2 n}}, \quad \tilde{C}=\text { const }, \forall(x, t) \in G_{i j}, \forall G_{i j} \in G .
$$

4. Итерационное уточнение аппроксимирующих полиномов. Полученное таким образом полиномиальное приближение искомой функции $u(x, t)$ используется для подстановки в правую часть вместо выражения этой функции, после чего снова строится полиномиальное приближение правой части, и описанные действия повторяются с применением соотношений (19)(21). Абстрактно предполагается, что количество итераций не ограничено. Данное построение интерполяции и итерационного уточнения выполняется в каждой подобласти $G_{i j}$ из (1)-(3) до завершения обхода всей области $G$. Приближение решения уравнения $(22)$ на текущей итерации получается путем аналитического интегрирования полинома из левой части (24) по времени в соответствии с (23) и по аналогии с (21). Приближения индексируются: ${ }^{t} P_{u, 2 k, 2 n, r}(x, t)$ означает приближение решения на текущей итерации, а ${ }^{t} P_{u, 2 k, 2 n,(r-1)}(x, t)-$ на предыдущей:

$$
{ }^{t} P_{u, 2 k, 2 n, r}(x, t)={ }^{t} P_{u, 2 k, 2 n, r}\left(x, t_{0}\right)+\int_{t_{0}}^{t}\left(f(x, t)-a(x, t) \frac{\partial^{t} P_{u, 2 k, 2 n,(r-1)}(x, t)}{\partial x}\right) d t+{ }^{t} c_{u, 2 k, 2 n}
$$

при этом $\partial^{t} P_{u, 2 k, 2 n,(r-1)}(x, t) / \partial x$ получается из ${ }^{t} P_{u, 2 k, 2 n,(r-1)}(x, t)$ по аналогии с $(20)$. Пусть зафиксировано число подобластей $2^{2 k}$ и пусть произвольно выбрана подобласть $G_{i j}$. Почленное 
вычитание равенств (23) и (26) влечет соотношение

$$
\begin{aligned}
u(x, t)-{ }^{t} P_{u, 2 k, 2 n, r}(x, t)=u\left(x, t_{0}\right) & -{ }^{t} P_{u, 2 k, 2 n, r}\left(x, t_{0}\right)+ \\
& +\int_{t_{0}}^{t}\left(-a(x, t) \frac{\partial}{\partial x}\left(u(x, t)-{ }^{t} P_{u, 2 k, 2 n,(r-1)}(x, t)\right)\right) d t-{ }^{t} c_{u, 2 k, 2 n} .
\end{aligned}
$$

Разность $u\left(x, t_{0}\right)-{ }^{t} P_{u, 2 k, 2 n, r}\left(x, t_{0}\right)$ представляет собой накопленную по предшествующим подобластям погрешность интерполирования, которая при итерационном уточнении в текущей подобласти не меняется, она присоединяется в качестве слагаемого ${ }^{t} c_{u, 2 k, 2 n}$, суммарная погрешность интерполяции (без учета итерационного уточнения в других подобластях) отмечается индексом $S$ :

$$
u(x, t)-{ }^{t} P_{u, 2 k, 2 n, r}(x, t)=\int_{t_{0}}^{t}\left(-a(x, t) \frac{\partial}{\partial x}\left(u(x, t)-{ }^{t} P_{u, 2 k, 2 n,(r-1)}(x, t)\right)\right) d t-{ }^{t} c_{S, u, 2 k, 2 n} .
$$

Для оценки погрешности (26) используется еще одно предположение:

V. Предполагается, что решение $u(x, t)$ задачи $(22)$ обладает следующим свойством:

$$
\left\{\begin{array}{l}
\forall \varepsilon>0 \exists \Delta=\Delta(\varepsilon): \forall \Delta x, 0<|\Delta x| \leqslant|\Delta|,\left|\frac{\partial u(x, t)}{\partial x}-\frac{u(x+\Delta x, t)-u(x, t)}{\Delta x}\right| \leqslant \varepsilon, \\
|\Delta|=\text { const } \forall(x, t), \quad(x+\Delta x, t) \in G .
\end{array}\right.
$$

Для оценки скорости сходимости вместо исходной задачи (22) с сохранением всех предположений будем рассматривать вспомогательную задачу:

$$
\frac{\partial u_{\Delta}}{\partial t}+a(x, t) \frac{\left(u_{\Delta}(x+\Delta, t)-u_{\Delta}(x, t)\right)}{\Delta}=f(x, t), \quad u_{\Delta}(x, 0)=\varphi(x),
$$

где $\Delta$ такое же, как в (27). В частности, для любого $\varepsilon>0$ найдется такое $\Delta=\Delta(\varepsilon)$, что

$$
\left|\frac{\partial u_{\Delta}}{\partial x}-\frac{u_{\Delta}(x+\Delta, t)-u_{\Delta}(x, t)}{\Delta}\right| \leqslant \frac{\varepsilon}{2}, \quad\left|\frac{\partial u}{\partial x}-\frac{u(x+\Delta, t)-u(x, t)}{\Delta}\right| \leqslant \frac{\varepsilon}{2},
$$

где значения $\Delta$, не умаляя общности, можно считать одинаковыми. Решение задачи (28) в каждой подобласти $G_{i j}$ будет приближаться аналогично тому, как изложено выше для задачи $(22),-$ полиномом ${ }^{t} P_{u, 2 k, 2 n, r}(x, t)$, который в данном случае обозначается ${ }^{t} \bar{P}_{u, 2 k, 2 n, r}(x, t)$. Значение

$$
f(x, t)-a(x, t) \frac{\left(u_{\Delta}(x+\Delta, t)-u_{\Delta}(x, t)\right)}{\Delta}
$$

будет приближаться полиномом $\Psi_{2 k, 2 n}^{i j}(z, w)$, который в этом приближении обозначается $\bar{\Psi}_{2 k, 2 n}^{i j}(z, w)$. Значения остаточных членов интерполяции изменятся, но их обозначения условно меняться не будут, что не должно приводить к недоразумениям. Точное решение задачи (28) примет следующий вид:

$$
u_{\Delta}(x, t)=u_{\Delta}\left(x, t_{0}\right)+\int_{t_{0}}^{t}\left(f(x, t)-a(x, t) \frac{u_{\Delta}(x+\Delta, t)-u_{\Delta}(x, t)}{\Delta}\right) d t .
$$

Приближенное решение этой задачи:

$$
\begin{aligned}
& { }^{t} \bar{P}_{u, 2 k, 2 n, r}(x, t)={ }^{t} \bar{P}_{u, 2 k, 2 n, r}\left(x, t_{0}\right)+ \\
& +\int_{t_{0}}^{t}\left(f(x, t)-a(x, t) \frac{{ }^{t} \bar{P}_{u, 2 k, 2 n,(r-1)}(x+\Delta, t)-{ }^{t} \bar{P}_{u, 2 k, 2 n,(r-1)}(x, t)}{\Delta}\right) d t+{ }^{t} c_{u, 2 k, 2 n}
\end{aligned}
$$


Отсюда

$$
\begin{aligned}
& u_{\Delta}(x, t)-{ }^{t} \bar{P}_{u, 2 k, 2 n, r}(x, t)=\int_{t_{0}}^{t}-a(x, t)\left(\frac{u_{\Delta}(x+\Delta, t)-{ }^{t} \bar{P}_{u, 2 k, 2 n,(r-1)}(x+\Delta, t)}{\Delta}-\right. \\
& \left.-\frac{u_{\Delta}(x, t)-{ }^{t} \bar{P}_{u, 2 k, 2 n,(r-1)}(x, t)}{\Delta}\right) d t-{ }^{t} c_{S, u, 2 k, 2 n,}, \\
& u_{\Delta}(x, t)-{ }^{t} \bar{P}_{u, 2 k, 2 n, r}(x, t)=\int_{t_{0}}^{t}\left(-a(x, t)\left(\frac{u_{\Delta}(x+\Delta, t)-{ }^{t} \bar{P}_{u, 2 k, 2 n,(r-1)}(x+\Delta, t)}{\Delta}-\right.\right. \\
& \left.\left.-\frac{u_{\Delta}(x, t)-{ }^{t} \bar{P}_{u, 2 k, 2 n,(r-1)}(x, t)}{\Delta}\right)-c_{S, 2 k, 2 n}\right) d t .
\end{aligned}
$$

Сначала рассматривается случай, когда существует такое постоянное $Q>0$, что

$$
0<\left|c_{S, 2 k, 2 n}\right| \leqslant Q \max _{G_{i j}}\left|u_{\Delta}(x, t)-{ }^{t} \bar{P}_{u, 2 k, 2 n,(r-1)}(x, t)\right|, \quad r=1,2, \ldots
$$

Пусть произвольно задано сколь угодно малое $\varepsilon>0$. Из (32) и (33) получим

$$
\begin{aligned}
& \left|u_{\Delta}(x, t)-{ }^{t} \bar{P}_{u, 2 k, 2 n, r}(x, t)\right| \leqslant \\
& \quad \leqslant \int_{t_{0}}^{t}\left(\frac{2 M}{\Delta} \max _{G_{i j}}\left|u_{\Delta}(x, t)-{ }^{t} \bar{P}_{u, 2 k, 2 n,(r-1)}(x, t)\right|+Q \max _{G_{i j}}\left|u_{\Delta}(x, t)-{ }^{t} \bar{P}_{u, 2 k, 2 n,(r-1)}(x, t)\right|\right) d t,
\end{aligned}
$$

где $M=\max _{G}|a(x, t)|=$ const. Можно считать, что $\Delta \leqslant 1$; с учетом произвольности выбора $(x, t) \in G_{i j}$ получим

$$
\max _{G_{i j}}\left|u_{\Delta}(x, t)-{ }^{t} \bar{P}_{u, 2 k, 2 n, r}(x, t)\right| \leqslant \frac{2 M+Q}{\Delta} \int_{t_{0}}^{t} \max _{G_{i j}}\left|u_{\Delta}(x, t)-{ }^{t} \bar{P}_{u, 2 k, 2 n,(r-1)}(x, t)\right| d t .
$$

Пусть $\varepsilon_{k, \ell}=\max _{G_{i j}}\left|u_{\Delta}(x, t)-{ }^{t} \bar{P}_{u, 2 k, 2 n, \ell}(x, t)\right| ;$ тогда

$$
\varepsilon_{k, \ell} \leqslant \frac{2 M+Q}{\Delta} \int_{t_{0}}^{t} \varepsilon_{k,(\ell-1)} d t, \quad \ell=1,2, \ldots
$$

При этом

$$
\varepsilon_{k, 0}=\max _{G_{i j}}\left|\bar{\varphi}\left(x, t_{j}\right)-{ }^{t} \bar{P}_{u, 2 k, 2 n, 0}(x, t)\right| \leqslant \max _{G_{i j}}\left|{ }^{t} c_{S, u, 2 k, 2 n}\right|,
$$

где $\bar{\varphi}\left(x, t_{j}\right)$ - функция, определяемая значением интерполяционного полинома на границе смежной подобласти $G_{i,(j-1)}$. Суммирование согласно $(25)$ по числу подобластей влечет

$$
\max _{G_{i j}}\left|{ }^{t} c_{S, u, 2 k, 2 n}\right| \leqslant \tilde{C} \frac{h^{2 n+1}}{2^{2 k n}}, \quad \tilde{C}=\text { const } .
$$

Отсюда и из (34) имеем

$$
\varepsilon_{k, 1} \leqslant \tilde{C} \frac{2 M+Q}{\Delta} \frac{h^{2 n+1}}{2^{2 k n}} \int_{t_{0}}^{t} d t ; \quad \Longrightarrow \quad \varepsilon_{k, 1} \leqslant \tilde{C} \frac{h^{2 n+1}}{2^{2 k n}} \frac{2 M+Q}{\Delta}\left(t-t_{0}\right) .
$$

С учетом (34) получим

$$
\varepsilon_{k, 2} \leqslant \tilde{C} \frac{h^{2 n+1}}{2^{2 k n}}\left(\frac{2 M+Q}{\Delta}\right)^{2} \frac{\left(t-t_{0}\right)^{2}}{2} .
$$


В продолжение итераций получится:

$$
\varepsilon_{k, \ell} \leqslant \tilde{C} \frac{h^{2 n+1}}{2^{2 k n}}\left(\frac{2 M+Q}{\Delta}\right)^{\ell} \frac{\left(t-t_{0}\right)^{\ell}}{\ell !}, \quad \ell=1,2, \ldots
$$

С учетом предположения I из (1)-(3) и (35) следует, что

$$
\varepsilon_{k, \ell} \leqslant \tilde{C} \frac{h^{2 n+1}}{2^{2 k n}}\left(\frac{2 M+Q}{\Delta} \cdot \frac{T}{2^{k}}\right)^{\ell} \frac{1}{\ell !}, \quad \ell=1,2, \ldots
$$

Поскольку правая часть стремится к нулю при любом основании степени с показателем $\ell$, то $\varepsilon_{k, \ell} \rightarrow 0, \ell \rightarrow \infty$. Вследствие этого

$$
\exists L_{\Delta}=L_{\Delta}(Q, \varepsilon): \max _{G_{i j}}\left|u_{\Delta}(x, t)-{ }^{t} \bar{P}_{u, 2 k, 2 n, \ell}(x, t)\right| \leqslant \frac{\varepsilon}{2} \quad \forall \ell \geqslant L_{\Delta} .
$$

Теорема 2. Для произвольно фиксированного числа подобластей $2^{2 k}$ в (3), при выполнении условия (33), последовательность итераций (32) для всех $(x, t) \in G_{i j}$ равномерно сходится $\kappa$ решению задачи (28) с оценками (36), (37) при любом выборе $i, j$ из (2).

Теорема 2 переносится (см. [12]) на общий случай без ограничения (33). Если считать, что уравнения (22) и (28) построены для одной и той же функции, то правая часть (28) изменится. С учетом этого изменения и на основе (29) можно показать (см. [12]), что соответствующее изменению приближение решения будет сколь угодно мало, равномерно в $G$, отклоняться от решения задачи (22): для любого $\varepsilon>0$ можно указать такие $\Delta>0$ и $L_{\Delta}=L_{\Delta}(Q, k, \varepsilon)$, что

$$
\max _{G}\left|u(x, t)-{ }^{t} \bar{P}_{u, 2 k, 2 n, \ell}(x, t)\right| \leqslant \tilde{C} \frac{h^{2 n+1}}{2^{k \times 2 n}}\left(\frac{2 M+Q}{\Delta} \cdot \frac{T}{2^{k}}\right)^{\ell} \frac{1}{\ell !} \leqslant \varepsilon \quad \forall \ell \geqslant L_{\Delta} .
$$

Аналогично строится приближение частных производных. Метод распространяется на нелинейное уравнение переноса. В [12] представлены схемы применения метода к уравнениям высших порядков, к системам уравнений в частных производных первого порядка, а также к интегродифференциальным уравнениям.

5. Численный эксперимент. Для задачи Коши

$$
\frac{\partial u}{\partial t}+\frac{\partial u}{\partial x}=-u+3 e^{t+x}, \quad u(x, 0)=e^{x}
$$

абсолютная погрешность предложенного решения $\left(h_{x} \approx 8 \cdot 10^{-2}, h_{t} \approx 4 \cdot 10^{-2}\right)$ сопоставляется с MathCAD (функция pdesolve; $h_{x}=10^{-2}, h_{t}=5 \cdot 10^{-3}$ ):

\begin{tabular}{|l|l|l|l|l|l|l|l|}
\hline$(x, t)$ & $(0,1 ; 1,0)$ & $(0,2 ; 1,0)$ & $\ldots$ & $(0,5 ; 1,0)$ & $\ldots$ & $(0,9 ; 1,0)$ & $(1,0 ; 1,0)$ \\
\hline MathСAD & $2,67 \cdot 10^{-3}$ & $1,48 \cdot 10^{-3}$ & $\ldots$ & $1,92 \cdot 10^{-4}$ & $\ldots$ & $3,13 \cdot 10^{-5}$ & $6,31 \cdot 10^{-7}$ \\
\hline $\begin{array}{l}\text { Предложенный } \\
\text { метод }\end{array}$ & $5,20 \cdot 10^{-18}$ & $1,52 \cdot 10^{-18}$ & $\ldots$ & $4,34 \cdot 10^{-19}$ & $\ldots$ & $3,90 \cdot 10^{-18}$ & $2,60 \cdot 10^{-18}$ \\
\hline
\end{tabular}

Аналогичные результаты получаются для набора других линейных и нелинейных уравнений переноса (см. [12]). Абсолютная погрешность приближения для отдельных задач в эксперименте росла до $3,14 \cdot 10^{-10}$, но оставалась ниже погрешности MathCAD, который давал $1,73 \cdot 10^{-4}$.

6. Заключение. Изложен компьютерный метод приближенного решения задачи Коши для уравнения переноса на основе кусочно-интерполяционной аппроксимации и итерационного уточнения. Интерполирующие решение и его частные производные полиномы Ньютона для функции двух переменных преобразованы к виду алгебраических полиномов с числовыми коэффициентами. На этой основе выполняется итерационное уточнение, которое представляет собой компьютерную реализацию приближений, аналогичных последовательным приближениям Пикара. Выполнен численный эксперимент, в котором достигается точность приближения решения линейного и квазилинейного уравнения переноса порядка $10^{-18}$ в единичной прямоугольной области. 


\section{СПИСОК ЛИТЕРАТУРЫ}

1. Березин И. С., Жидков Н. П. Методы вычислений. Т. 2. - М.: Физматгиз, 1962.

2. Голиков $A$. Н. Кусочно-полиномиальные схемы вычисления функций двух переменных, частных производных и двойных интегралов на основе интерполяционного полинома Ньютона/ Деп. в ВИНИТИ 20.09.2010, № 528-B2010. - Таганрог: Таганрог. ин-т имени А. П. Чехова (филиал), 2010.

3. Голиков A. Н. Моделирование электрон-фононного рассеяния в нанопроволоках на основе кусочнополиномиального приближения функций двух переменных с минимизацией временной сложности/ дисс. на соиск. уч. степ. канд. техн. наук. - Таганрог: ЮФУ, 2012.

4. Джануни, Г. А., Ромм Я. Е. Варьируемое кусочно-интерполяционное решение задачи Коши для обыкновенных дифференциальных уравнений с итерационным уточнением// Ж. вычисл. мат. мат. физ. 2017. - 57, № 10. - C. 1641-1660.

5. Жамбалова Д. Б., Черный С. Г. Метод интерполяционного профиля решения уравнений переноса// Вестн. НГУ. Сер. Информ. технол. - 2012. - 10, № 1. - С. 33-54.

6. Калиткин Н. Н. Численные методы. - М.: Наука, 1978.

7. Лебедев A. С., Черный С. Г. Практикум по численному решению уравнений в частных производных. - Новосибирск: Изд-во Новосиб. гос. ун-та, 2000.

8. Макаров Е. Г. Инженерные расчеты в Mathcad 15. - СПб.: Питер, 2011.

9. Рогов Б. В., Михайловская М. Н. Монотонная высокоточная компактная схема бегущего счета для квазилинейных уравнений гиперболического типа// Мат. модел. $-2011 .-23$, № 12 . - С. 65-78.

10. Ромм Я. Е. Локализация и устойчивое вычисление нулей многочлена на основе сортировки, II// Киберн. сист. анал. - 2007. - № 2. - С. 161-174.

11. Ромм Я. Е., Голиков А. Н. Распараллеливаемые кусочно-полиномиальные схемы аппроксимации функций, производных и вычисления определённых интегралов с повышенной точностью/ Деп. в ВИНИТИ 27.04.2010, № 230-В2010. - Таганрог: Таганрог. ин-т имени А. П. Чехова (филиал), 2010.

12. Ромм Я. Е., Джсануни Г. А. Оценки скорости сходимости кусочно-интерполяционного решения задачи Коши с итерационным уточнением для уравнения переноса/ Деп. в ВИНИТИ 12.02.2018, № 20-В2018. - Таганрог: Таганрог. ин-т имени А. П. Чехова (филиал), 2018.

13. Самарский А. А. Теория разностных схем. - М.: Наука, 1989.

14. Сиковский Д. Ф. Методы вычислительной теплофизики. - Новосибирск: Изд-во Новосиб. гос. ун-та, 2013.

15. Тихонов A. Н., Самарский A. А. Уравнения математической физики. - М.: Наука, 1977.

16. Barth T. J., Deconinck H. (eds.) High-Order Methods for Computational Physics. — Berlin: Springer, 1999.

17. Biermann O. Über näherungsweise Kubaturen// Monats. Math. Phys. - 1903. - 14. - P. 211-225.

18. Gasca M., Sauer T. On the history of multivariate polynomial interpolation// J. Comput. Appl. Math. 2000. - 122. - P. 23-35.

19. Liu X.-D., Osher S. Nonoscillatory high order accurate self-similar maximum principle satisfying shock capturing schemes, I// SIAM J. Numer. Anal. — 1996. — 33, № 2. — P. 760-779.

Ромм Яков Евсеевич

Таганрогский институт им. А. П. Чехова (филиал)

Ростовского государственного экономического университета

E-mail: romm@list.ru

Джанунц Гарик Апетович

Таганрогский институт им. А. П. Чехова (филиал)

Ростовского государственного экономического университета

E-mail: janunts@inbox.ru 